\title{
Model-based electrospinning process design for nanofibers production
}

\author{
Marlena Drąg ${ }^{1, *}$, Pawet Drąg ${ }^{1, *}$ \\ ${ }^{1}$ Wrocław University of Science and Technology, Department of Control Systems and Mechatronics, Wrocław, Poland
}

\begin{abstract}
The main concept of the electrospinning process modeling is to overcome difficulties associated with the experimental-based optimization procedure. Thus, 3-dimensional polynomial models were discussed in this work. Electrospinning process modeling is an approach that supplies the information about wanted concentrations of the electrospinning solution components and will also make it possible to obtain bead-free nonwovens with desired fiber diameter. The application area for described methodology is highly associated with the environmental field of study, where the design of the functionalized nanofibers production is extremely requested (e.g. separation, filtration and purification processes).
\end{abstract}

\section{Introduction}

Electrospinning is greatly comprehensive method by which production of nanofibers is possible. Nanofibers can be formed from different polymers, polymer blends or composites [1]. The schematic diagram of the electrospinning setup is presented on the Fig. 1.

Nanofibers fabricated via electrospinning process exhibit unique properties, especially very large surface area to volume ratio, high pore density and design flexibility for physical or chemical functionalization $[2,3]$. Due to this fact, researchers concentrate their effort to develop the new application areas for electrospun nanofibers $[1,4]$. For more than a decade, the strongest increases in production of the electrospun nanofibers was observed in the tissue engineering, wound dressing and drug delivery application sectors $[5,6]$. Nowadays electrospun nanofibers are especially promising candidates to be used in the environmental protection field of study [7].

There are many research papers that have reveal promising results of combining very high nanofibers surface area and complexation ability of cyclodextrins (CDs) $[2,3,7]$. Cyclodextrines are cyclic oligosaccharides produced by enzymatic conversion of starch. By forming non covalent host-guest complexes they can entrap variety of organic molecules, including volatile organic compounds (VOCs). Cyclodextrins can exist in the three forms: $\alpha, \beta$ and $\gamma[3,8,9]$.

One of the most promising solutions for obtaining functionalized electrospun nonwovens dedicated to the phenolphthalein removal is production of the polystyrene (PS) nanofibers containing $\beta$-cyclodextrin $(\beta-C D)$ [2]. In turn, nonwovens for aniline incarceration can be yielded through the electrospinning of $\gamma$-cyclodextrin $(\gamma$-CD) using a small amount of polyethylene oxide (PEO) [3]. An interesting solution also seems to be electrospinning of the hydroxypropylbeta-cyclodextrin (HP $\beta C D)$ and hydroproxypyl-gammacyclodextrin $(\mathrm{HP} \gamma \mathrm{CD})$ for entrapment tests of aniline and benzene [7].

According to the literature the most essential structural characteristic of the electrospun nanofibers web is fiber diameter [5]. Additionally, to produce unique structures without any beads along the fibers length is still challenging. Needles to say, that electrospinning process require complex optimization procedure, because it may be affected by many parameters (e.g. polymer solutions parameters, processing and ambient conditions). For this reason scientists have practical cause for creation of the electrospinning process mathematical models [1,5]; models that can describe obtained results, determine unmeasured set of characteristics or explain process behavior [10-13].

Mathematical models that have been used in the literature usually consist of two important modeling zones. The first one is associated with the capillary and the second one with the whipping instability area $[5,14]$. With regard to the consideration of forces the electrospinning process flow path model can be presented as follows

$$
m \frac{d^{2} p}{d t^{2}}=f_{C}+f_{E}+f_{V}+f_{S}+f_{A}+f_{G}+\ldots
$$

where

$m$ - equivalent mass,

$P$ - position coordinate,

$f_{C}$ - Coulombic forces,

$f_{E}$ - electric field forces,

$f_{V}$ - viscoelastic forces,

$f_{s}$ - surface tension forces, 
$f_{A}$ - air drag forces,

$f_{G}$ - gravitational forces.

In particular the mentioned forces can be described by physical relations

$$
f_{C}=\frac{e^{2}}{l^{2}}
$$

$f_{\bar{E}}=-\frac{e V_{0}}{h}$,

$$
\frac{d \sigma_{V}}{d t}=\frac{G}{l} \frac{d l}{d t}-\frac{G}{\mu} \sigma_{V_{e}}
$$$$
f_{s}=\frac{\alpha \pi R^{2} k}{\sqrt{x^{2}+y^{2}}}[i|x| \operatorname{sign}(x)+j|y| \operatorname{sign}(y)],
$$

$f_{A}=0.65 \pi R \rho_{\text {air }} v^{2}\left(\frac{2 v R}{v_{\text {air }}}\right)^{-0.81}$

$f_{G}=\rho g \pi R^{2}$,

where

$e$ - charge,

$l$ - length of ideal rectilinear jet,

$V_{0}$ - applied voltage,

$h$ - drop-collector distance,

$\sigma_{V}$ - viscoelastic stress,

$G$ - elastic modulus,

$\mu$ - viscosity,

$\alpha$ - surface tension coefficient,

$\mathrm{R}$ - jet radius,

$k$ - jet curvature,

$\rho$ - density,

$v$ - kinematic viscosity [1].

It is worth to say, that mathematical models mentioned in the literature have diverse incompleteness level, which not necessarily denotes they are poor or useless. Thus, it seems that introduced simplifications can improve the calculations and help to focus on the specific issue of the electrospinning process [1].

In this work we have been focused on the experimental data confrontation with the mathematical models. This way may stand an effective approach to the practical tool development for the electrospinning process design.

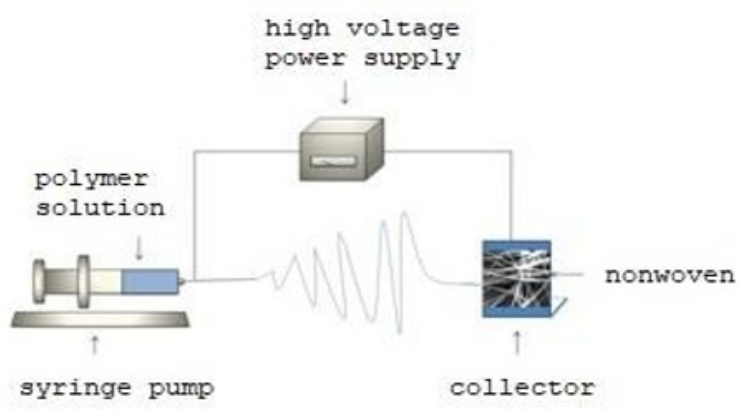

Fig. 1. The schematic diagram of the electrospinning process.

\section{Methodology}

\subsection{Problem formulation}

This work is aimed at the new approach to the electrospinning process design of the functionalized polystyrene nanofibers. The presented approach may be useful in the fulfilling the lack of regular and completely described experimental observations, as well as theoretical features necessary in controlling effective results obtained. The complex physical relations have not been considered in this work; presented methodology is associated with the process parameters and measured results relations. All measurements and their detailed description used in this work have been shown in the research of Uyar et al. [2].

As it is commonly known, the electrospinning process may be characterized by the set of input parameters, that reflect process and ambient conditions [1]. In the considered task the following assumptions were taken into account

Assumption 1. The ambient conditions are constant and the process parameters take the following values:

- feed flow rate $=1 \mathrm{ml} / \mathrm{h}$,

- applied voltage $=15 \mathrm{kV}$,

- tip-to-collector distance $=10 \mathrm{~cm}$,

- needle inner diameter $=0.4 \mathrm{~mm}$.

Assumption 2. There are two parameters, that have been designed in this work: $\beta$-cyclodextrin $(\beta-\mathrm{CD})$ and polystyrene (PS) concentration.

The mentioned PS concentration was varied from $10-25 \%$ (w/v, with respect to the solvent) and the $\beta-\mathrm{CD}$ concentration was varied from $1-50 \%(\mathrm{w} / \mathrm{w}$, with respect to the polymer).

Assumption 3. The PS/ $\beta-C D$ nonwoven is the result of the electrospinning process.

Assumption 4. The obtained nonwoven can be characterized by two parameters: fiber diameter [nm] and beads width $[\mu \mathrm{m}]$. 
As it was mentioned before, the main essential structural characteristics of the obtained fabric is the fiber diameter [5]. It is very important to obtained unique, bead-free nonwovens. Structural nonwoven characteristic can be determined by using specialist devices, e.g. scanning electron microscope (SEM), transmission electron microscope (TEM) or atomic force microscope (AFM). In this work the fiber size and beads width have been determined from scanning electron microscope images $[1,2,5]$.

\subsection{Solution procedure}

The presented methodology is based on the experimental data confrontation with the cause and effect models, where the fiber diameter/beads width depend on the PS and $\beta$-CD concentration

$$
\begin{aligned}
& F_{d}=f_{1}(P S, \beta C D) \\
& B_{w}=f_{2}(P S, \beta C D),
\end{aligned}
$$

where

$F_{d}$ - fiber diameter [nm],

$B_{w}$ - beads width $[\mu \mathrm{m}]$,

PS - polystyrene,

$\beta C D$ - $\beta$-cyclodextrin,

and

$$
\begin{aligned}
& f_{1}: \mathbb{R}^{2} \rightarrow \mathbb{R}, \\
& f_{2}: \mathbb{R}^{2} \rightarrow \mathbb{R} .
\end{aligned}
$$

Based on the adopted assumptions and experiment results the triplets of the following form have been chosen

$$
\begin{aligned}
& m_{F d, i}=\left(P S_{i}, \beta C D_{i}, F_{d i}\right), \\
& m_{\bar{b}, i}=\left(P S_{i}, \beta C D_{i}, B_{w i}\right),
\end{aligned}
$$

where

$m_{F d, i}-$ the fiber diameter measurement values,

$m_{\bar{b}, i}-$ the beads width measurement values, for $\mathrm{i}=1, \ldots, 15$.

Then, to describe obtained data two threedimensional models were proposed

- linear

$$
y_{2}=A x+d_{1}
$$

- quadratic

where

$$
y_{Q}=x^{\tau} B x+C x+d_{2} \text {, }
$$

$$
\begin{aligned}
& x=\left[\begin{array}{c}
P S \\
\beta C D
\end{array}\right], \\
& A=\left[a_{1}, a_{2}\right],
\end{aligned}
$$

$$
\begin{aligned}
& B=\left[\begin{array}{ll}
b_{1} & b_{2} \\
b_{3} & b_{4}
\end{array}\right], \\
& C=\left[c_{1}, c_{2}\right],
\end{aligned}
$$

and $a_{1}, a_{2}, b_{1}, b_{2}, b_{3}, b_{4}, c_{1}, c_{2}, d_{1}, d_{2} \in \mathbb{R}$.

The values of the unknown parameters were found according to the least squares method

-for the fiber diameter models

$$
\begin{aligned}
& \sum_{i=1}^{15}\left(y_{L F_{d, i}}-m_{F d, i}\right)^{2} \rightarrow \min , \\
& \sum_{i=1}^{15}\left(y_{Q F_{d, i}}-m_{F d, i}\right)^{2} \rightarrow \min ,
\end{aligned}
$$

- for the beads width models

$$
\sum_{i=1}^{15}\left(y_{L B_{W i} i}-m_{B w_{i} i}\right)^{2} \rightarrow \min ,
$$

$\sum_{i=1}^{15}\left(y_{Q \bar{B}_{W i} i}-m_{\bar{B} w_{i} i}\right)^{2} \rightarrow \min$.

Then, the most common metrics: Mean Absolute Error (MAE), Mean Squared Error (MSE) and Root Mean Squared Error (RMSE) were used to measure model accuracy

$$
\begin{aligned}
& M A E=\frac{1}{n} \sum_{i=1}^{n}\left|y_{i}-m_{i}\right|, \\
& M S E=\frac{1}{n} \sum_{i=1}^{n}\left(y_{i}-m_{i}\right)^{2}, \\
& \text { RMSE }=\sqrt{M S E} .
\end{aligned}
$$

\section{Results and discussion}

As the result of the carried out research we obtained four cause and effect models. Two of them were related to the fiber diameter, the other two to the beads width. The unknown model parameters were specified as follows

$$
\begin{gathered}
y_{L F_{d}}=\left[\begin{array}{ll}
118.12 & 23.34
\end{array}\right] x-1197.6 \\
y_{Q F_{d}}=x^{\tau}\left[\begin{array}{ll}
7.80 & 2.48 \\
2.48 & 0.33
\end{array}\right] x+[-153.46-46.53] x \\
+939.64
\end{gathered}
$$




$$
\begin{gathered}
y_{L \bar{W}}=[-0.29-0.13] x-9.62, \\
y_{Q B_{W}}=x^{T}\left[\begin{array}{cc}
-0.059 & -0.019 \\
0.019 & -6 \cdot 10^{-4}
\end{array}\right] x+ \\
+[1.770 .33] x-6.33 .
\end{gathered}
$$

Linear and quadratic model equations for fiber diameter indicate some information useful from the process design point of view. First of all the fiber diameter is growing according to the growing PS and $\beta$-CD concentration. Moreover, it can be observed that fiber diameter and PS concentration relationship should be modeled by nonlinear equations, whereas fiber diameter/ $\beta$-CD concentration dependence is almost linear. In turn, the beads width is decreasing according to the growing PS and $\beta$-CD concentration. Relationship between beads width and $\beta-C D$ seems to be linear.

The fitting results of the designed models to the experimental data are presented in the Table 1 and Table 2 for the fiber diameter and beads width models, respectively. It is worth to highlight, that fiber diameter for obtained, experimental data is in the range ca. 150-2000 nm. For the linear model of the fiber diameter the MAE value is equal to 134 . Therefore, the value of MAE is comparable with the measured fiber diameter, especially for the lower values. For the quadratic model of the fiber diameter the MAE value is equal to 50. That difference does not influence the obtained result significantly. As the result of the carried out research it was observed, that MSE and RMSE values are smaller for the quadratic models of fiber diameter.

The beads width is in the range ca. 3-8 nm. MAE values are 1.40 and 0.96 for linear and quadratic models, respectively. Thus, we can say that obtained MAE values for both models are similar. However, MAE value for quadratic model does not change the obtained result significantly.

According to the performed computations we can say that both, linear and quadratic models can give an insight into main cause and effect relations, and characterize the nonlinearity of observed dependences. Described models have been used in aim to experimental data interpolation. At the current stage extrapolation issue is not considered.

The presented models are useful to fulfill the absence of systematic, completely characterized experimental observations [1]. Furthermore using mathematical models can meaningly reduce the experimental time and costs by determining the most probable set of process conditions. The proposed approach indicates desired concentrations of the electrospinning solution compounds ( $\beta-C D$ and PS) and enables us to obtain nonwovens with desired fiber diameter and beads as small as possible. Model-based information can significantly improve the electrospinning process design and can be used for the functionalized nanofibers production.
Table 1. Fiber diameter models accuracy.

\begin{tabular}{cccc}
\hline Model type & MAE & MSE & RMSE \\
\hline Linear $y_{2}$ & 134 & 25376 & 159 \\
& & & \\
Quadratic $y_{Q}$ & 50 & 4733 & 69 \\
\hline
\end{tabular}

Table 2. Beads width models accuracy.

Model type MAE MSE RMSE

\begin{tabular}{cccc} 
Linear $y_{2}$ & 1.40 & 2.79 & 1.67 \\
Quadratic $y_{Q}$ & 0.96 & 1.38 & 1.17 \\
\hline
\end{tabular}

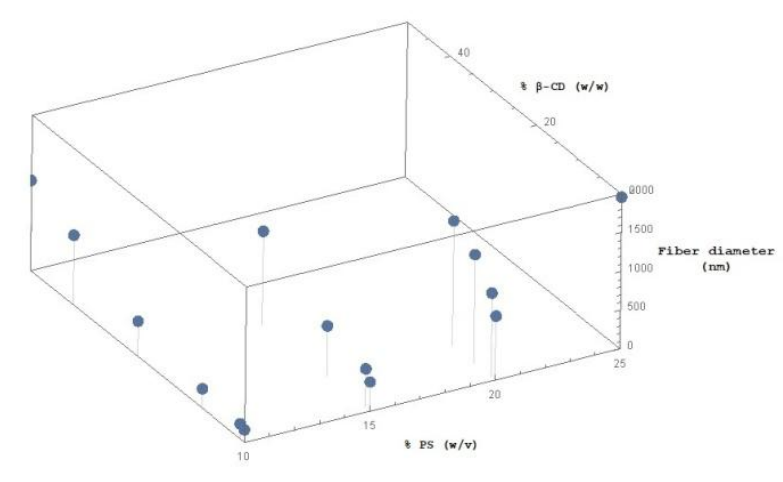

Fig. 2. The fiber diameter experimental data results. 


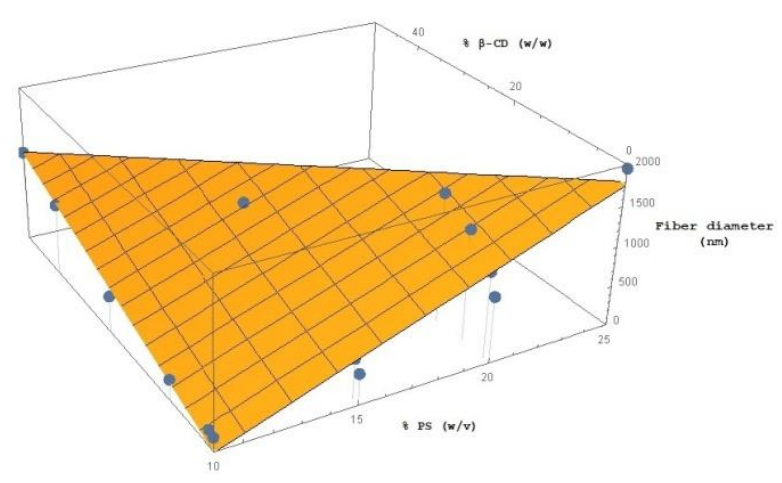

Fig. 3. The fiber diameter and $\beta-\mathrm{CD} / \mathrm{PS}$ concentration relationship; linear model.

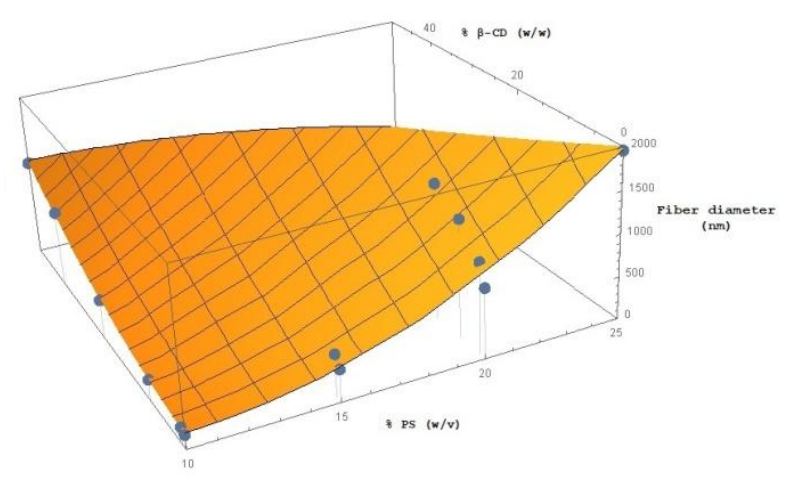

Fig. 4. The fiber diameter and $\beta-\mathrm{CD} / \mathrm{PS}$ concentration relationship; quadratic model.

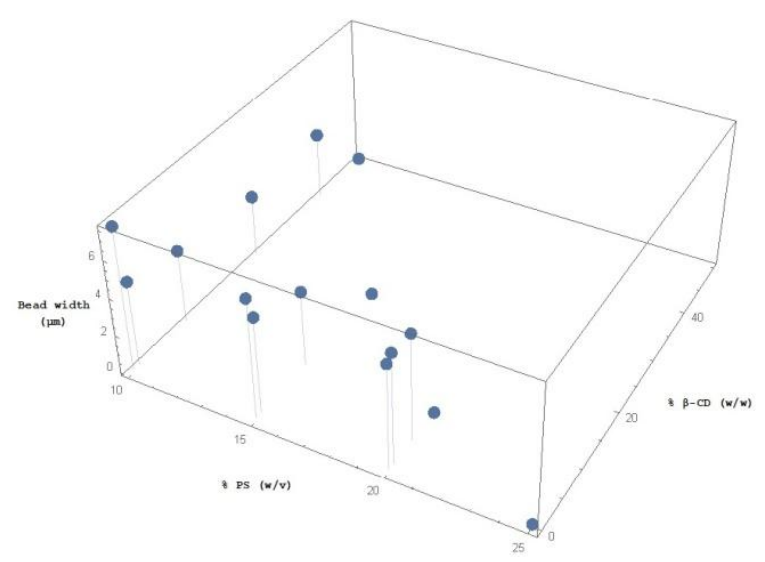

Fig. 5. The beads width experimental data results.

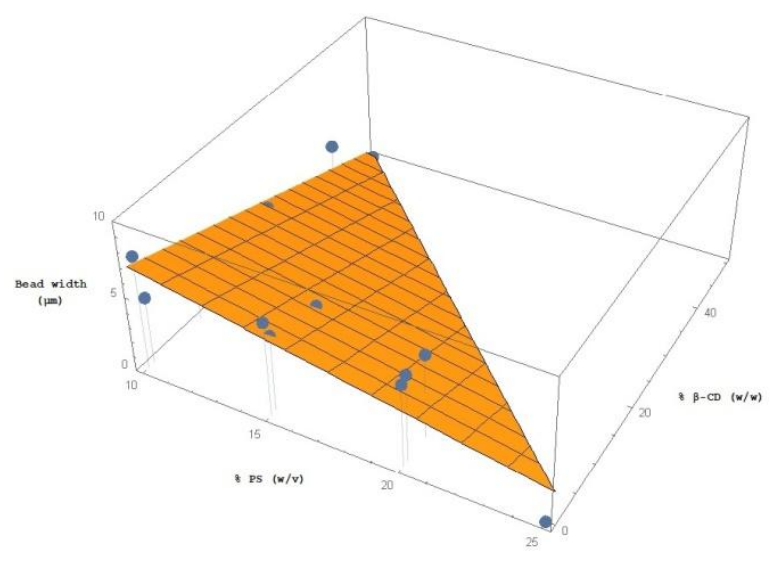

Fig. 6. The beads width and $\beta-\mathrm{CD} / \mathrm{PS}$ concentration relationship; linear model.

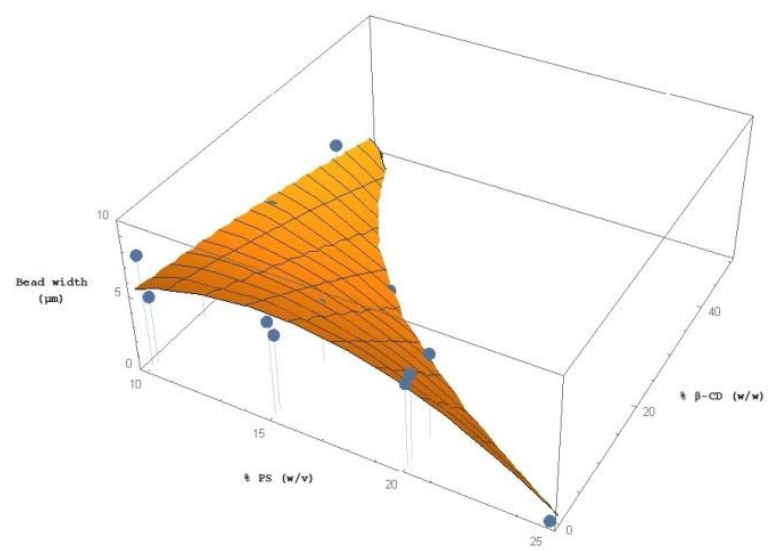

Fig. 7. The beads width and $\beta-\mathrm{CD} / \mathrm{PS}$ concentration relationship; quadratic model.

\section{Conclusions}

The experimental way of the electrospinning process design is often cost- and time-consuming. The uniform fibers production needs advanced knowledge based on the process specified experience. It is worth to say, that each experiment is related with electrospinning solution preparation, nanofibers formation and the fibers characterization by using sophisticated devices, (e.g. scanning electron microscope (SEM) or transmission electron microscope (TEM)). To overcome these difficulties and facilitate tedious optimization procedure model-based design approach was presented in this work. Thus, 3-dimensional polynomial models were discussed. To describe a chosen experimental relations meet in the nanofibers production, two polynomial models (linear and quadratic) were proposed. The experimental data were used to the model parameters optimization. As a result, the reasonable set of parameters was indicated, as well as the dependencies between fiber diameter/beads width and $\beta-\mathrm{CD} / \mathrm{PS}$ concentration were considered. Although the quadratic model resulted with better fitting features, the linear model also can be used to improve an experimental 
procedure design. The proposed methodology points out desired concentrations of the electrospinning solution components and will also make it possible to obtain bead-free nonwovens with certain fiber diameter.

As it was indicated, some specified nanofibers features can be described using multidimensional polynomial models, that can be applied in the real-life experimental procedures. The proposed models allow us to gather fully characterized specification of desired fibers production. Moreover, due to a small amount of required parameters they are very convenient, whereas definitely bigger set of information is required (e.g. jet radius, jet curvature, surface tension coefficient, etc.) when solving models based on the Newton's Second Law of motion [1].

The presented in this work 3-dimensional polynomial models may stand an effective approach to the practical tool development for the electrospinning process design associated with the environmental field of study, where cyclodextrin functionalized nanofibers production is extremely required (e.g. filtration, separation and purification processes) [2]. What is more presented models may be useful not only in the production of the functionalized nanofibers, but also in the other electrospinning processes leading to the structures of diverse morphology and characteristics.

\section{Acknowledgements}

This work was supported by the grant 0401/0146/17 at Department of Control Systems and Mechatronics, Wrocław University of Science and Technology.

\section{References}

1. S. Ramakrishna et al., An Introduction to Electrospinning and Nanofibers (WSPC, Singapore, 2005)

2. T. Uyar et al., Nanotechnology 20 (2009)

3. A. Celebioglu et al., J. Incl. Phenom.Macro 90, 135141 (2018)

4. Kenry et al., Prog. Polym. Sci. 70, 1-17 (2017)

5. S. Rafiei et.al., Cell. Chem. Technol. 47, 323-338, (2013)

6. L. Weng et al., Curr. Pharm. Design. 21 (2015)

7. A. Celebioglu et al., Chemosphere 144, 736-744 (2016)

8. E. M. Martin Del Valle, Process. Biochem. 39, 1033-1046 (2004)

9. E. Gatica et al., J. Photoch. Photobio. A. 348, 295-304 (2017)

10. L.T. Biegler, Nonlinear Programming: Concepts, Algorithms, and Applications to Chemical Processes (SIAM, Philadelphia, 2010)

11. J.T. Betts, Practical Methods for Optimal Control and Estimation Using Nonlinear Programming (SIAM, Philadelphia, 2010)
12. L.T. Biegler, et al., Control and Optimization with Differential-Algebraic Constraints (SIAM, Philadelphia, 2012)

13. J. Nocedal et al., Numerical Optimization (Springer, Heidelberg, 2006)

14. A.E. Senador Jr. et al., Mat. Res. Soc. Symp. Proc. 661, KK5.9.1-KK5.9.6 (2001) 